\title{
A Novel Lipid System Forming Hollow Microtubes at High Yields and Concentration
}

\author{
Jean-Paul Douliez, ${ }^{*}$, \\ Cédric Gaillard ${ }^{2}$ \\ Laurence Navailles and Frédéric Nallet ${ }^{3}$
}

1 : INRA/BIA, équipe Interface et Systèmes Dispersés, rue de la Géraudière, F-44316 Nantes, France

2 : INRA/BIA, plate-forme microscopie, rue de la Géraudière, F-44316 Nantes, France

3 : Centre de Recherche Paul Pascal, CNRS, 115 Av. Albert Schweitzer, F-33600 Pessac, France

* corresponding author: email: jean-paul.douliez@ nantes.inra.fr

Phone: + 33240675083

RECEIVED DATE (to be automatically inserted after your manuscript is accepted if required according to the journal that you are submitting your paper to) 


\section{Supporting information}

Material and methods

\section{Phase contrast microscopy}

Observations were made at room temperature at 20x magnification using an optical microscope in the phase contrast mode (Nikon Eclipse E-400, Tokyo, Japan) equipped with a 3-CCD JVC camera

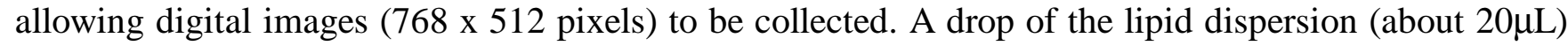
was deposited on the glass slide surface $(76 \times 26 \times 1.1 \mathrm{~mm}, \mathrm{RS}$ France) and covered with a cover slide (22x22 mm, Menzel-Glaser, Germany). The glass slides were previously cleaned with ethanol and acetone.

Small-angle neutron scattering (SANS)

Small-angle neutron scattering (SANS) experiments were performed at Laboratoire LéonBrillouin (laboratoire mixte CEA/CNRS, Saclay, France) on spectrometer PAXY. The neutron beam was collimated by appropriately chosen neutron guides and circular apertures, with a beam diameter at the sample of $7.6 \mathrm{~mm}$. The neutron wavelength was set to 4 or $8 \AA$ with a mechanical velocity selector $(\Delta \lambda / \lambda \approx 0.1)$, the $2 \mathrm{D}$ detector $\left(128 \times 128\right.$ pixels, pixel size $\left.5 \times 5 \mathrm{~mm}^{2}\right)$ being positioned at 1.4 or $6.7 \mathrm{~m}$, respectively. The scattering wave vector, $Q$, then ranges from typically 0.006 to $0.5 \AA^{-1}$, with a significant overlap between the two configurations. The samples, prepared with deuterated water, were held in flat quartz cells with a $2 \mathrm{~mm}$ optical path and temperature-controlled by a circulating fluid to within $\pm 0.2^{\circ} \mathrm{C}$. The azimuthally-averaged spectra were corrected for solvent, cell and incoherent scattering, as well as for background noise.

\section{Transmission Electron Microscopy}


Because of the viscosity and the concentration, samples did not freeze properly and cryo-TEM could not be performed. However, we obtained interesting images from a sample which was deposited on the TEM grid, dried and then frozen in liquid nitrogen. The freezing procedure allowed to break the lipid tubes and reveal their inner structure. The present images show a lipid tube, the upside of which has been broken upon freezing (fig. 1 left, arrow). Although only 3 membranes can be observed (fig. 1, right) and although a shrinkage has probably occur because of the experimental TEM conditions, a lamellar repeat distance of about 30nm can be measured in good agreement with our SANS data.

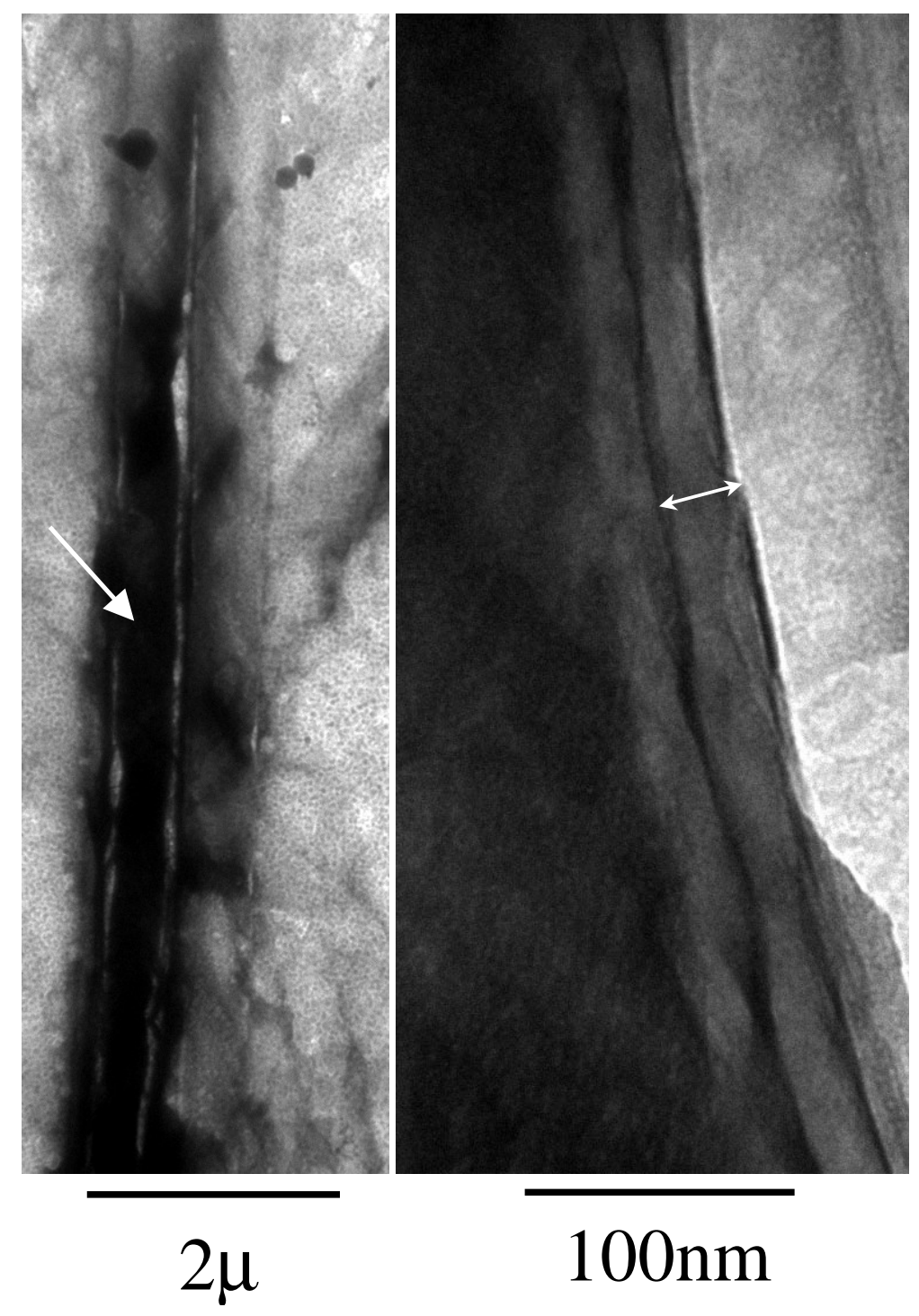

Figure 1. TEM images of a frozen sample after drying on the TEM grids. 